\title{
The ADAQ framework: An integrated toolkit for data acquisition and analysis with real and simulated radiation detectors
}

\author{
Zachary S. Hartwiga,* \\ ${ }^{a}$ Department of Nuclear Science and Engineering and the Plasma Science and Fusion \\ Center, MIT, Cambridge MA 02139, USA
}

\section{Abstract}

The ADAQ framework is a collection of software tools that is designed to streamline the acquisition and analysis of radiation detector data produced in modern digital data acquisition (DAQ) systems and in Monte Carlo detector simulations. The purpose of the framework is to maximize user scientific productivity by minimizing the effort and expertise required to fully utilize radiation detectors in a variety of scientific and engineering disciplines. By using a single set of tools to span the real and simulation domains, the framework eliminates redundancy and provides an integrated workflow for high-fidelity comparison between experimental and simulated detector performance. Built on the ROOT data analysis framework, the core of the ADAQ framework is a set of $\mathrm{C}++$ and Python libraries that enable high-level control of digital DAQ systems and detector simulations with data stored into standardized binary ROOT files for further analysis. Two graphical user interface programs utilize the libraries to create powerful tools: ADAQAcquisition handles control and readout of real-world DAQ systems; and ADAQAnalysis provides data

\footnotetext{
${ }^{*}$ Corresponding author: hartwig@mit.edu, +1 (617) 253-5471
} 
analysis and visualization methods for experimental and simulated data. At present, the ADAQ framework supports digital DAQ hardware from CAEN S.p.A. and detector simulations performed in Geant4; however, the modular design will facilitate future extension to other manufacturers and simulation platforms.

Keywords: Data acquisition and analysis, Radiation detection, Waveform digitization, Detector readout, Detector simulation PACS: 07.05.Hd, 07.05.Tp, 29.50.+v, 29.85.-c,

1 1. Introduction to the $A D A Q$ framework

2 The relatively recent proliferation of digital data acquisition (DAQ) sys3 tems for radiation detectors has provided significant advances over tradi4 tional analog DAQ systems. In addition to the replacement of large, bulky, 5 and expensive analog hardware with digital algorithms, advantages include 6 preservation of unshaped detector signals, implementation of digital pulse 7 processing (DPP) algorithms that are not possible or difficult in analog cir8 cuits, unlimited optimization of DPP algorithms in post-processing, and sig9 nal stability against gain drifts or increased noise from analog shaping circuits that are no longer needed [1]. Furthermore, due to technological advances

\footnotetext{
${ }^{1}$ CAEN website: http://www.caen.it/csite

${ }^{2}$ Struck website: http://struck.de/
} 
such as nuclear security, nuclear fission and fusion energy, medical physics, ion beam materials analysis, accelerator-based science, and undergraduate education in the teaching laboratory.

The ability to rapidly and seamlessly integrate digital DAQ hardware into the research workflow requires the availability of complementary high performance software. This is particularly important for commodity users, who, unlike the particle and nuclear physics communities, often have far less resources and expertise than are necessary to implement high quality in-house software. These users utilize detectors and DAQ systems as standard laboratory tools rather than as a core component of their research. To meet this need, the ADAQ framework - a set of software libraries and graphical user interface (GUI) programs designed to provide production-quality acquisition and analysis of radiation detector data with digital DAQ systems - is being developed. Furthermore, ADAQ is built on top of two world-leading software projects that significantly enhance its capabilities: ROOT [2], a scientific software framework that provides data processing, statistical analysis, visualization, and persistent storage; and Geant4 [3], a Monte Carlo particle transport toolkit for the comprehensive simulation of particles interacting with matter. The ADAQ framework is open source, cost-free, modular, and extensable, providing the user with a powerful and accessible solution for experimental and simulated data acquisition with radiation detectors.

The purpose of this paper is impart to the reader a high level description of the tools in the ADAQ framework and an understanding of their performance capabilities in a modern digital DAQ system. In order to do so, this paper is structured as follows: Section 2 describes how the ADAQ frame- 

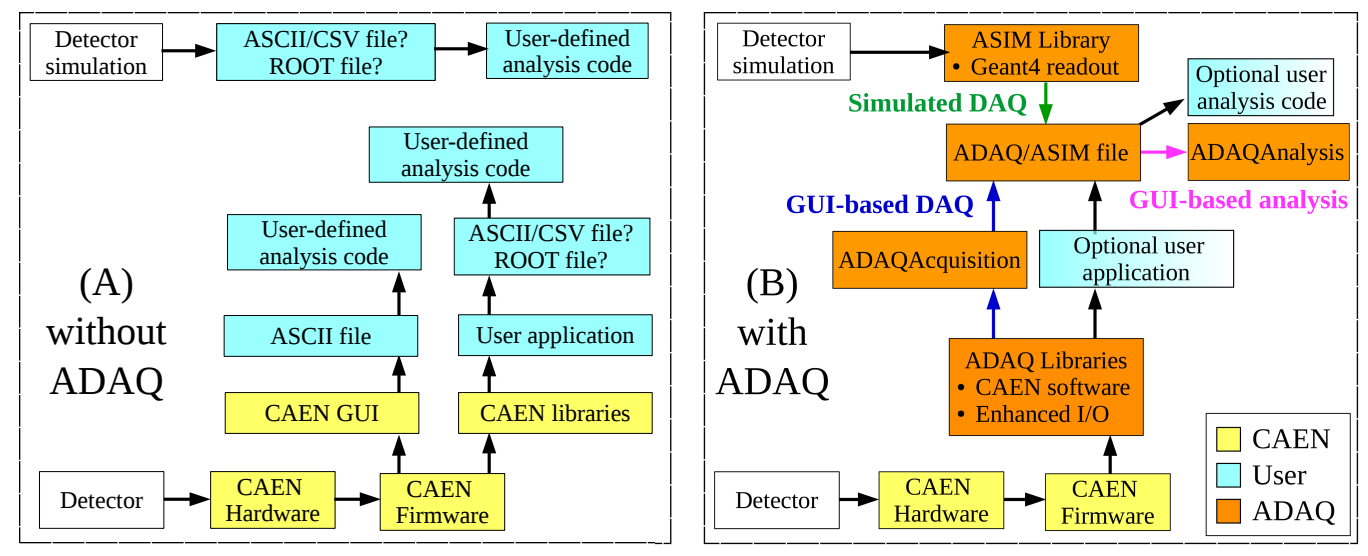

Figure 1: Two examples of workflows that incorporate radiation detector data acquisition and analysis. (A) Without ADAQ, the real-world and simulated detector workflows are completely separate with substantial work and expertise required by the user despite tools provided by digital DAQ manufacturers. (B) With ADAQ, the workflows are integrated together to provide fidelity between experiment and simulation with substantial reduction in required user effort and expertise.

work meets some of the challenges in modern radiation detection; Section 3 describes the ADAQ software libraries and file formats; Section 4 describes ADAQAcquisition, a program for control, readout, and data storage with digital DAQs; Section 5 describes ADAQAnalysis, a program for offline analysis of experiment and simulated detector data; and finally, Section 6 presents some concluding remarks.

\section{Overview of the ADAQ framework}

\subsection{Challenges in detector data acquisition and simulation}

As described in the introduction, fully utilizing the power of digital DAQ hardware requires complementary software to acquire, readout, and analyze detector data. While high performance digital DAQ hardware is becoming 
more affordable, widespread, and available directly from commercial vendors (rather than developed in-house), acquisition and analysis software has largely not kept pace. Most vendors of high-performance digital DAQ systems designed for detector applications, such as CAEN or Struck, specialize in digital DAQ hardware and only offer a limited set of software development and production tools that in many instances do not meet the need of many users who require high quality software tools. Researchers must either divert substantial effort and resources from their primary science or engineering research into implementing their own software or in the absence of software and DAQ expertise simply forego the inclusion of digital DAQs in their experiments.

Another area where digital DAQs can be transformative is in the teaching laboratory. Historically, students have used traditional analog DAQ systems, which require expertise in analog circuits and electronics to understand the fundamentals of pulse processing, or performed acquisition and analysis with commercial "black box" software, which often obfuscates the underlying operations on detector pulses. Digital DAQs, in contrast, alleviate both of these issues. By acquiring raw, unprocessed digital waveforms and then analyzing the pulses with simple digital algorithms that can be easily written by the student, digital DAQs enable the student to focus on the most critical aspects of detector data acquisition and analysis fundamentals while simultaneously being exposed to the systems that are increasingly prevalent in the research environment.

This primary challenge in digital DAQ is depicted graphically in Figure 1A. The figure shows common experiment and simulation workflows us- 
ing available hardware and software from CAEN. The first challenge is the amount of effort and expertise required of the user as indicated by the dominance of cyan-colored boxes in the flow chart. Demands are placed on the user such as implementation of data file structures, analysis codes for simulation and experiment, and from-scratch data acquisition software. Such efforts can take substantial resources (time, money, personnel) away from the scientific or engineering goals and are obvious tasks that could be commoditized for the researcher. However, while CAEN provides a GUI program to control their digitizers, they are intended for demonstration purposes and are not suitable for production-level experiments. CAEN provides a fairly comprehensive set of low-level software libraries, but these require substantial software development and data acquisition expertise to fully utilize.

A second challenge that is evident from the figure is the decoupling of the workflows between real-world and simulated detectors. This has the affect of increasing the user workload due to implementation of multiple data formats and analysis codes while lowering the fidelity between experiment and simulation since the data is not necessarily processed with the same set of algorithms.

\subsection{The ADAQ framework solution}

As shown in Figure 1B, the ADAQ framework is composed of three primary tools that empower the researcher by providing a comprehensive and integrated software environment that enables the high performance acquisition and analysis of radiation detector data and that unites the experiment and simulation workflow for high-fidelity results. At the core of the framework is the ADAQ and ASIM libraries, shown at the bottom and top, respec- 
tively of the figure. The ADAQ libraries [4] are a set of $\mathrm{C}++$ and Python libraries that provide a standard interface between firmware running on DAQ hardware and a computer with data stored in binary ROOT files known as ADAQ files; the ASIM libraries provide a standard interface between Geant4 simulated detectors and the computer with data stored in binary ROOT files known as ASIM files. The ADAQAcquisition [5] program utilizes the ADAQ libraries to create a GUI-based program to control digital DAQs and automatically read out data into ADAQ files. Finally, the ADAQAnalysis [6] program provides a GUI-based program for comprehensive analysis of data that is stored in ADAQ and ASIM files, giving the user a complete toolchain for the acquisition and analysis of detector data from hardware or simulation. In addition, if the expert user wishes to implement his or her own custom applications for specialized needs, the ADAQ libraries and ADAQ/ASIM file formats provide a standardized, enhanced, and more powerful starting point for development compared to vendor provided software tools.

The three ADAQ tools described above are available as open source, cost-free tools to the user for download or collaboration via GitHub under the GNU G.P.L. v3.0, which gives the user flexibility to copy, modify, and redistribute the codes to best serve his or her research needs. As of December 2015, the ADAQ framework is compatible with the most recent versions of ROOT (version 6.06.00) and Geant4 (version 10.02). The code and documentation is under active development, expanding the acquisition and analysis capabilities and the range of hardware on which the ADAQ tools can be run. The ADAQ framework is presently used in a number of experiments across several institutions, including an accelerator-based diagnostic for materials 
inside the Alcator C-Mod tokamak at the MIT Plasma Science and Center [7], a multiple monoenergetic gamma radiography experiment for nuclear security involving MIT, Penn State, and Georgia Tech [8], and detector development on a novel scintillator-based neutron detector at Penn State [9]. The ADAQ framework is also being used in teaching laboratories on radiation and detectors within the Department of Nuclear Science and Engineering at MIT.

\section{The ADAQ libraries}

The ADAQ libraries are a collection of $\mathrm{C}++$ and Python libraries that provide a modular software interface to digital DAQ hardware. The purpose of these libraries is to provide a set of standardized, hardware-agnostic, object-oriented libraries that not only wrap the low-level libraries provided by hardware manufacturers but extend them to perform more powerful, higherlevel functionality. These libraries provide an excellent foundation for the ADAQAcquisition (Section 4) and ADAQAnalysis (Section 5) programs but also for programmers who write their own acquisition and analysis code.

At present, the ADAQ libraries only support digital DAQ hardware from CAEN, including the following units:

- VME bridge: The V1718 VME-USB bridge board

- High voltage: The V6533 and V6534 families of VME high voltage boards

- Waveform digitizers: The x720, x724, x725, x730, x751, both in VME board and desktop form factors 
- High voltage + digitizer: DT5790 desktop unit

The ADAQ libraries, however, have been designed such that future support for hardware from other vendors is easily accommodated by adding new software classes for each new piece of hardware that defines the hardware register maps, integrates vendor software libraries, and implements specific methods to control the hardware through the ADAQ libraries.

\subsection{The $A D A Q$ and ASIM file formats}

The use of efficient, standardized format data files is at the core of the ADAQ framework. Ensuring the data is stored in a consistent and comprehensive format streamlines the connection between data readout and data analysis, provides a uniform data interface for different analysis methods, and eliminates redundancy of having separate data formats for real-world and simulated detectors. The ADAQ framework relies on the highly compressed binary ROOT file to accomplish this. The ROOT file enables the persistent storage of a wide range of data types, from fundamental data types to complex classes, and is capable of massive and efficient data input/output. Rigidly ensuring that ROOT files are written from any ADAQ tool with a prescribed set of data objects guarantees a standard file format for analysis, either using an ADAQ framework tool or using custom-analysis code developed by the user. For ROOT files storing real-world detector data, the files are known as ADAQ files and indicated as such by the .adaq.root extension. Similarly, for ROOT files storing simulated detector data, the files are known as ASIM files and indicated as such by the .asim.root extension. 


\subsection{The ADAQControl library}

The ADAQControl library is composed of three related classes all inheriting from the common, pure abstract ADAQVBoard class that provides a generic set of descriptors for a DAQ device, such as hardware identifiers, VME address, and connection state of the device. Each of the three derived classes ADAQDigitizer, ADAQHighVoltage, and ADAQBridge - provide a generic programming interface to a range of hardware within the digitizer, high voltage, and VME bridge device types. Each class provides methods to control all aspects of the hardware, from reading/writing individual hardware register values to more complex functions, such as powering high voltage channels or reading out digitized waveforms.

\subsection{The ADAQReadout library}

The ADAQReadout library is composed of a manager class, ADAQReadoutManager class, that provides the user with an easy way to create and populate specially formatted, compressed binary ROOT TFiles - known as ADAQ files (See Section 3.1) - with data acquired through the digital DAQ control methods contained in the ADAQControl library. Raw digitized waveforms are stored as $\mathrm{C}++$ vectors in ROOT TTree data objects within the ADAQ File. Two supporting classes act as standardized containers for the data: the ADAQWaveformData class holds event-level waveform data (baseline, pulse area, pulse height, PSD integrals, time stamp, channel ID, and device ID); the ADAQReadoutInformation holds run-level information on the DAQ hardware and a complete record of hardware, firmware, and software settings used in data acquisition. 


\subsection{The ASIM libraries}

The ASIM libraries provide a conduit through which detector data simulated with Geant4 can be stored within specially formatted, compressed binary ROOT TFiles - known as ASIM files (See Section 3.1) - and analyzed with the ADAQAnalysis program. This gives the user convenient access to the same powerful data analysis methods (e.g. spectra calibration, continuum calculation, fitting, integrating) that are used in analyzing real detector data stored in ADAQ files. This capability provides a streamlined and highfidelity approach to comparing real-world and simulated detectors.

An example is shown in Figure 2, in which ADAQAnalysis was used to compare experimental and simulated $14.1 \mathrm{MeV}$ detector response functions. The experimental setup was a $5.1 \phi \times 5.1 \mathrm{~cm}$ EJ301 liquid organic scintillator coupled to a 2-inch PMT and placed $1 \mathrm{~m}$ in front of a $14.1 \mathrm{MeV}$ neutron generator. The detector was read out by a CAEN V1720 VME digitizer board running standard firmware. The V1720 was located in a CAEN VME8100 powered crate and controlled by ADAQAcquisition through the PC via the CAEN V1718 USB-VME bridge board located in slot 0 of the crate. Detector data was stored in an ADAQ file for offline analysis in ADAQAnalysis. The Geant4 simulation setup was identical to the experimental geometry with the simulated detector read out through the ASIM libraries with data stored in an ASIM file for offline analysis in ADAQAnalysis.

Two libraries are created. The first - the ASIMReadoutManager library handles the readout of data from the user's Geant4 simulation. The library includes a set of special Geant4-derived sensitive detector and hit classes that the user can incorporate into his/her own simulation. Event-level informa- 


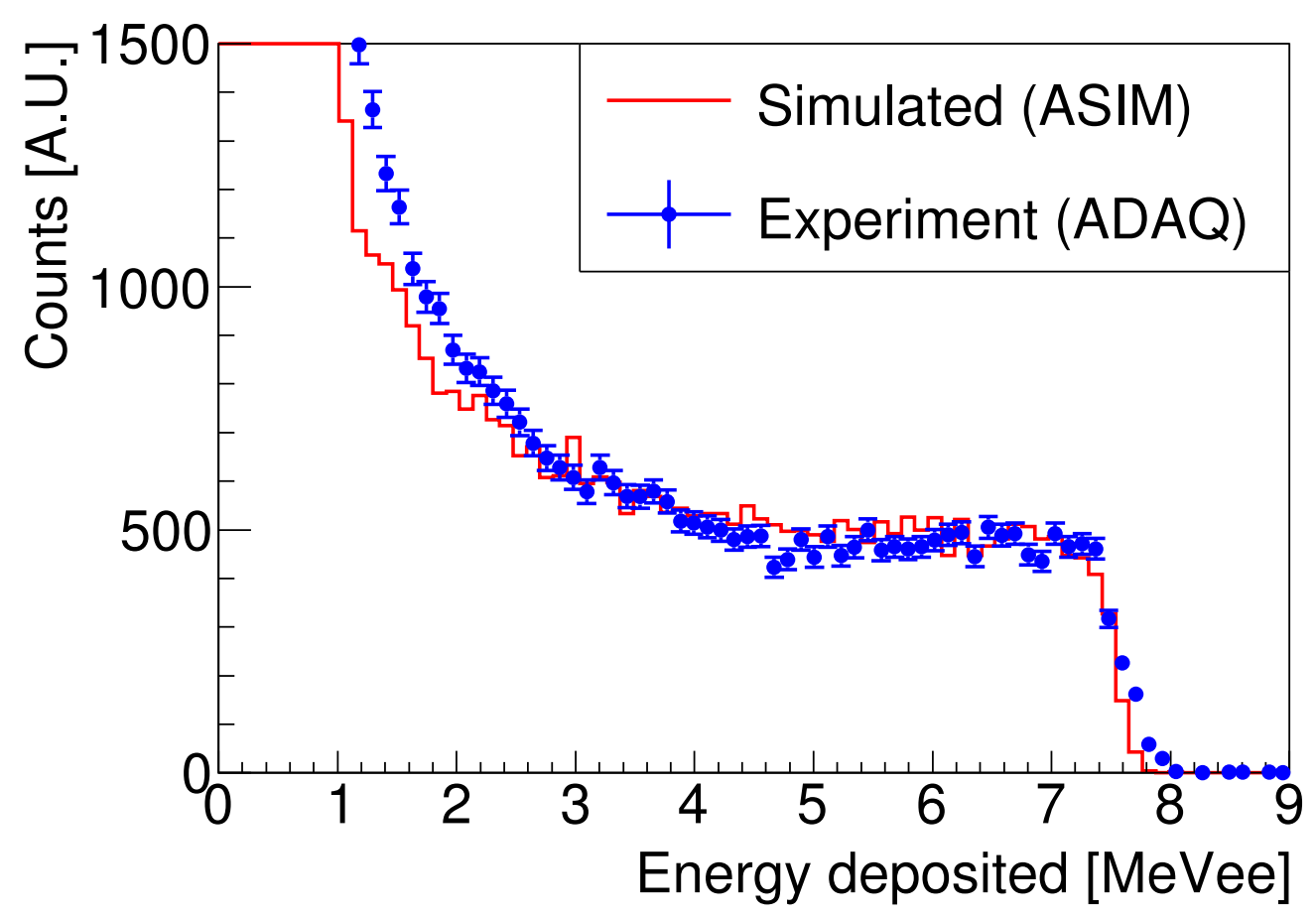

Figure 2: A comparison between experiment and simulated detector response of an EJ301 liquid organic scintillator detector to $14.1 \mathrm{MeV}$ neutrons. The experimental data was acquired with ADAQAcquisition and CAEN digitizers; the simulated data was produced with an ASIM-integrated Geant4 simulation that utilizes recent capabilities of the Geant4 toolkit to simulate photon production from nonlinear scintillators [10]. Both data sets were calibrated and histogrammed identically using ADAQAnalysis, which ensures high fidelity when comparing experimental and simulated detector data. 
tion - stored in ASIMEvent class objects - can include total energy deposition, optical photon production, and optical photon detection in a photodetector such as a photomultiplier tube or silicon photomultiplier. Run-level information - stored in ASIMRun class objects - includes incident particles, detected particles, optical photons created, and optical photons detected, with a set of methods to allow the user to specify thresholds for scoring events. The library is capable of handling readout in parallel processing through the MPIManager class, which provides the user an easy way to parallelize a Geant4 simulation. The second library - the ASIMStorageManager library - handles the persistent storage to ASIM files on disk the information that is read out with the ASIMReadoutManager library methods.

ASIM functionality is intentionally split between two libraries in order to isolate the dependence on Geant4 headers and libraries from the ASIMStorageManager library. This enables users to build the ADAQAnalysis program and analyze ASIM files on systems without Geant4 installed. Example simulations are available within the distribution to show the user how to integrate the ASIM libraries into his or her own Geant4 simulation.

\section{ADAQAcquisition: DAQ hardware control and readout}

ADAQAcquisition is a GUI-based program that implements control, acquisition, readout, and persistent storage for digital DAQ systems. The program provides the user with several levels of control over the hardware, from low-level tasks such as individual register read/write operations to more complex high-level tasks such as acquiring calibrated energy spectra or pulse shape discrimination (PSD) histograms in real-time. In addition to a fully 


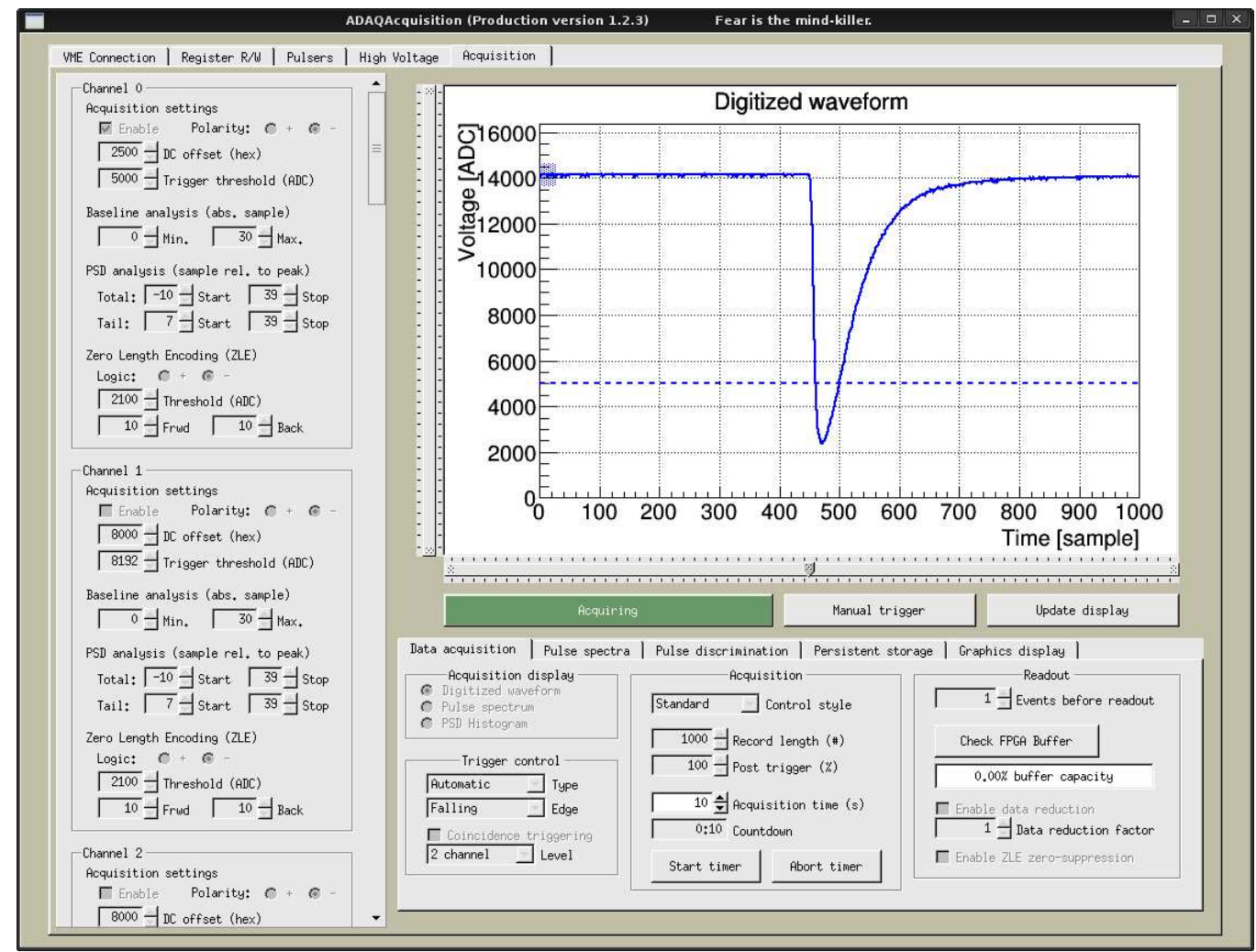

Figure 3: ADAQAcquisition acquiring a waveform from a cosmic ray in digital oscilloscope mode. The detector was a $10.16 \mathrm{~cm}$ cube of $\mathrm{NaI}(\mathrm{Tl})$ scintillator read out by a 3 -inch PMT and digitized with a CAEN V1725 VME board in a CAEN 8100 VME crate.

featured digital oscilloscope and multichannel analyzer, the program provides persistent storage of waveform data in ADAQ files for offline analysis, either with the user's own analysis code or with the ADAQAnalysis program described in Section 5. Other useful features are packaged in as well, such as an acquisition timer, checks on system dead time and data loss, and high-quality graphical output. The digital oscilloscope in ADAQAcquisition is shown in Figure 3 displaying a cosmic ray waveform from a $\mathrm{NaI}(\mathrm{Tl})$ detector.

As discussed in Section 3, ADAQAcquisition is at present only compati- 
ble with the hardware from CAEN that is supported by the ADAQ libraries; however, as support is added for new hardware from other vendors into the ADAQ libraries as described in Section 3, the ADAQAcquisition graphical interface, hardware programming, and readout methods can easily be updated.

\subsection{DAQ hardware programming}

ADAQAcquisition is designed to provide control over three main types of DAQ hardware: PC-to-VME bridge boards, high voltage units, and waveform digitizers. It enables this control through a GUI, which gives the user complete high-level control of all aspects of the hardware. In addition, direct reading and writing of individual hardware registers is possible for low-level control, with translation and graphical display of binary, decimal, hexadecimal numbers used in such programming enhancing usability.

The first type of supported hardware is the PC-to-VME bridge boards that form a standard interface between the boards running in a VME enclosure and the PC that controls them. These boards are typically located in slot 0 of a VME crate and connected to the PC either via a USB cable or an optical fiber. The program provides methods to interface with any digitizer and high voltage board attached to the VME backplane via the VME bridge by specifying the address in VME space; control of other features found on CAEN VME bridge boards, such as digital signal generators, are also provided.

The second type of supported hardware is high voltage units typically used to power radiation detectors. Upon connection to a high voltage unit, either a VME board via the VME bridge or a desktop high voltage unit, the 
program will automatically configure the user interface for the specific unit, providing the correct number of channels, proper safety limits on voltage and current, and initializing the board in a safe, powered off state. The user has the ability to set maximum voltage and current trip points, as well as to monitor the active voltage and drawn current in real time.

The third type of supported hardware is waveform digitizers used to read out radiation detectors signals. Upon connection to a digitizer, the ADAQAcquisition interface is automatically configured not only for the hardware but also for the type of digitizer firmware. ADAQAcquisition supports standard CAEN firmware for all digitizer families and desktop units as well the digital pulse processing pulse shape discrimination (DPP-PSD) firmware that runs on CAEN's x720, x725, and x730 families of digitizers as well as the DT5790 desktop unit. The user has the ability to set all global and channel-specific settings for digitizer control and readout, as well as on-board FPGA processing in the case of DPP-PSD firmware. Future upgrades to the ADAQ framework will include support for CAEN's DPP-CI (charge integration) and DPP-PHA (pulse height analysis) firmware on supported digitizer hardware.

\subsection{Waveform digitizer readout}

Following digitizer programming, readout of digitized information occurs once the user has initiated acquisition and started to accumulate data. In detector acquisition, data is usually packaged into "events", which typically contain the information acquired during a single trigger of the system. Events in ADAQAcquisition can include event metadata (e.g. event number, trigger time stamp, the organization and byte structure the event), the full digitized waveform, and the results of processing the data on the digitizer FPGA (e.g. 
baseline, pulse area, pulse height). For digitizer triggering, ADAQAcquisition provides three methods: manual triggering via a user action, automatic channel self-triggering when the digitized signal exceeds a threshold, or external triggering using a standardized TTL or NIM signal input on the digitizer front panel. In self-triggering mode, the user may set a coincidence level requirement on triggering, from requiring coincidence between two channels up to all channels on the digitizer units. The user may specify how many triggers occur (i.e. how many events are allowed to accumulate in digitizer memory) before the data is transferred from the digitizer to the PC for further processing.

ADAQAcquisition completely handles the transfer of events to the PC using the proprietary software protocols of the digitizer manufacturer over USB or optical cables. For example, on CAEN digitizers using standard firmware, an aggregate of events that includes the entire digitized waveform is transferred, where the proprietary structure of the events are decoded into usable data. On CAEN digitizers running advanced firmware, such as DPPPSD, the user's may choose which information to include in the event - full waveforms, FPGA-processed data, or both - before transfer and decoding, providing the ability to optimize data format and throughput. Once the events have been transferred from the digitizer, the data can be optionally further processed on the PC CPU. These actions can include waveform analysis (e.g. calculating baselines, pulse areas, pulse heights, and PSD integrals), and creation of energy spectra or pulse shape analysis histograms to examine particle discrimination or pileup status using the dual charge or dual gate method. 


\subsection{Live analysis and persistent storage}

ADAQAcquisition provides two primary real-time analysis methods. The first is to act a full feature digital oscilloscope, providing instant graphical feedback on the acquired waveforms and various acquisition settings. The user has an interactive graphical display that can be zoomed for detailed inspection, custom configured to best show the data, and saved in many image formats. The second is to act as much more powerful digital MCA, providing real-time pulse spectra with optional energy calibration as well as pulse shape analysis histograms to examine particle discrimination or pileup status using the dual charge or dual gate method.

The user has several options for saving data persistently to disk. The user may store any combination of full waveforms, analyzed waveform data (timestamp, baseline, pulse area, pulse height), or PSD integrals depending on his/her post-processing needs in an ADAQ file; the format and contents of the ADAQ file are described in Section 3.1. Data storage can be enabled/disabled automatically via an acquisition time or manually by user control. An energy acceptance window from the pulse spectra can be used as a filter to ensure only waveforms meeting certain criteria are stored to disk. Finally, the user has the option of saving the pulse spectra and PSD histograms directly to disk, either as ROOT objects in a ROOT file or as raw text in an ASCII or CSV formatted file. 


\section{ADAQAnalysis: analysis of experimental and simulated detec- tor data}

ADAQAnalysis is a GUI-based program that provides powerful analysis methods for experimental and simulated radiation detector data that is stored in ADAQ or ASIM file formats. For real detectors, data is most easily produced using the ADAQAcquisition program described in detail in Section 4 although the user can build his or her own data acquisition application using the ADAQ libraries in order to utilize the standard ADAQ file format required by ADAQAnalysis. For simulated detectors, such data is produced by integrating the ASIM libraries into the user's Geant4 detector simulation as described in Section 3.4. A screenshot of ADAQAnalysis is shown in Figure 4 performing PSD on waveforms from a EJ301 liquid organic scintillator detector.

\subsection{Waveform inspection and processing configuration}

To utilize the power of waveform digitization, ADAQAnalysis provides a set of waveform inspection and analysis tools that can be applied to either real or simulated waveforms stored in an ADAQ or ASIM file, respectively. These tools enable the user to examine waveforms in detail and then optimize the full set of parameters that is used in processing the waveforms into energy spectra or pulse shape discrimination histograms.

1. Baseline calculation: A time window can be specified in any part of the waveform in which the pulse baseline will be calculated. Functioning as a digital baseline restoration circuit, this feature maximizes the accuracy of pulse height and area calculation in signals with moderate 


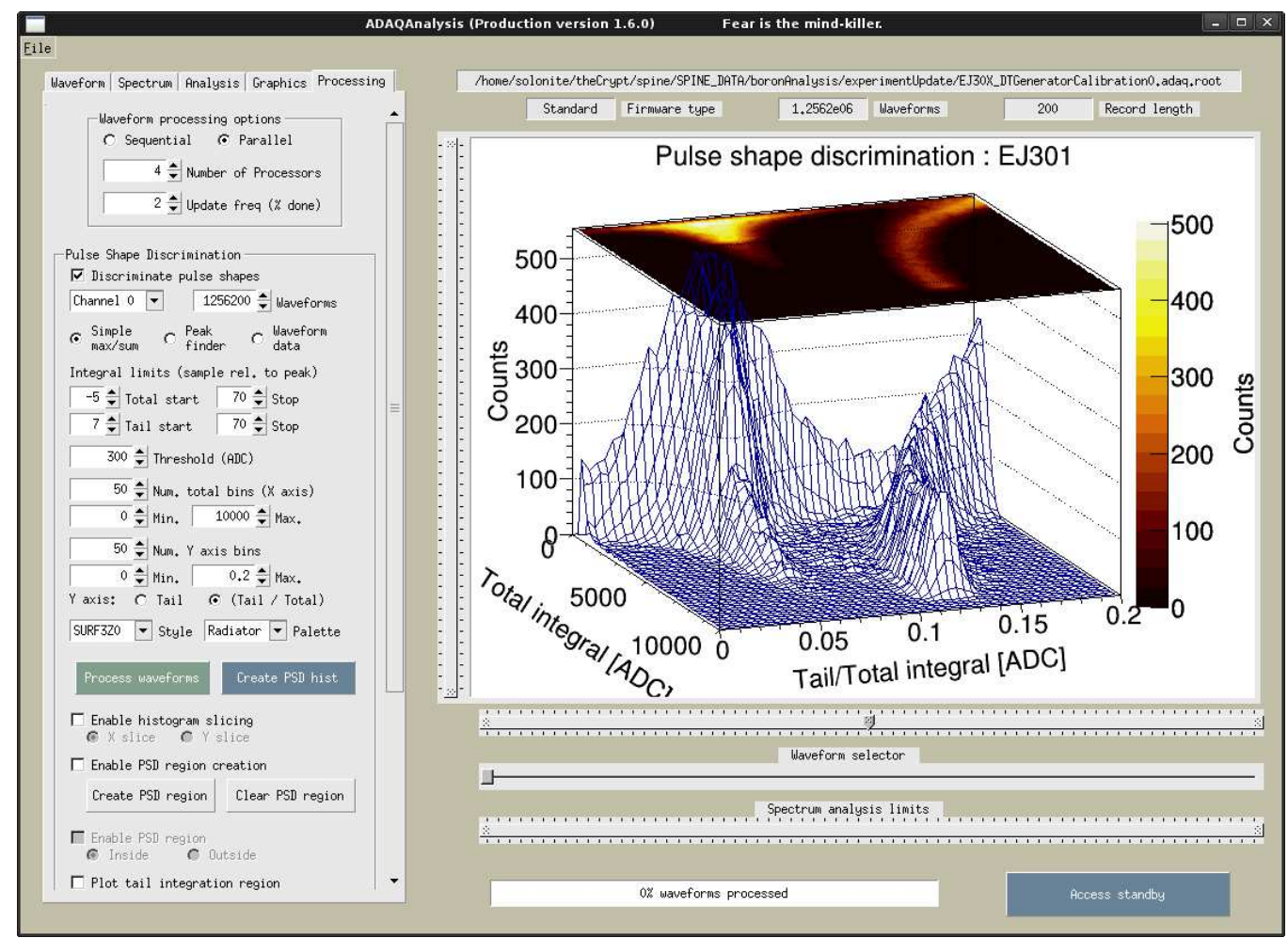

Figure 4: ADAQAnalysis performing pulse shape discrimination on neutron and gamma events induced by a $14.1 \mathrm{MeV}$ fast neutron source. The detector was a $2.5 \phi \times 2.5 \mathrm{~cm}$ EJ301 liquid organic scintillator read out by a 1-inch PMT and digitized with a CAEN V1720 VME board in a CAEN 8100 VME crate. 
noise of slowly drifting baselines relative to the pulse timescale. The user can plot a baseline-subtracted waveform as they adjust the time window to see the impact on the resulting waveform.

2. Zero suppression: Waveform samples below a user-specified threshold will be removed from the waveform before processing, eliminating very small amplitude data and maximizing computation efficiency without sacrificing accuracy. Known as zero suppression, this technique is particularly useful for long waveforms in which many peaks appear in a single acquisition window. The user can plot a zero-suppressed waveform as they adjust the zero-suppression threshold to see the impact on the resulting waveform.

3. Multipeak algorithm: In some cases, it is either advantageous or unavoidable to acquire a single acquisition window with many peaks. Examples may include pulsed accelerator systems where a trigger signal may be used to open an acquisition window during the beam pulse or acquisition of waveforms at extremely high rates, such that postprocessing pileup deconvolution algorithms may be implemented. Utilizing the sensitive nonlinear iterative peak-clipping (SNIP) algorithm [11], ADAQAnalysis provides a robust, tuneable peak-finding algorithm that can locate and calculate individual peak heights/areas for energy spectroscopy or pulse shape rejection algorithms. A demonstration of the algorithm appears in Figure 5, in the algorithm is clearly able to identify all peaks in the long acquisition window.

4. Pileup rejection: Waveforms that overlap in time, or pile up, can be 
identified and removed during waveform processing to prevent contaminating an energy spectrum or pulse shape discrimination histogram. With pileup rejection enabled, the user can inspect waveforms to see which peaks are accepted and rejected, an example of which is shown in Figure 5. The double peak that occurs around 1000 ns is flagged by the algorithm for rejection and shaded red; the remaining well-separated peaks are accepted by the algorithm and shaded green.

Once waveform processing parameters have been set, as described in Section 5.1, waveforms can be processed into either pulse spectra or PSD histograms with two algorithms. The first - the "max/sum" algorithm - is a simple but computationally fast algorithm that rapidly finds the maximum pulse height or sums samples into a pulse area within a user-selected waveform time range. The second algorithm - the "peak finder" algorithm - is more computationally intensive but provides greater waveform analysis capabilities, including identifying many peaks in a single acquisition.

\subsection{Energy spectra creation and analysis}

One of the primary features of ADAQAnalysis is the ability to process detectors waveforms into spectra. For real detectors storing data in ADAQ files, spectra can be created for any digitizer channel as either pulse height or pulse area spectra. For simulated detectors storing data in ASIM files, spectra can be created for any registered ASIM readout from energy deposited, number of optical photons created per event, or number of optical photons detected per event, as described in Section 3.4.

For uncalibrated spectra, the user can locate calibration points in two ways: either locating spectral peaks - suitable for many types of detectors 


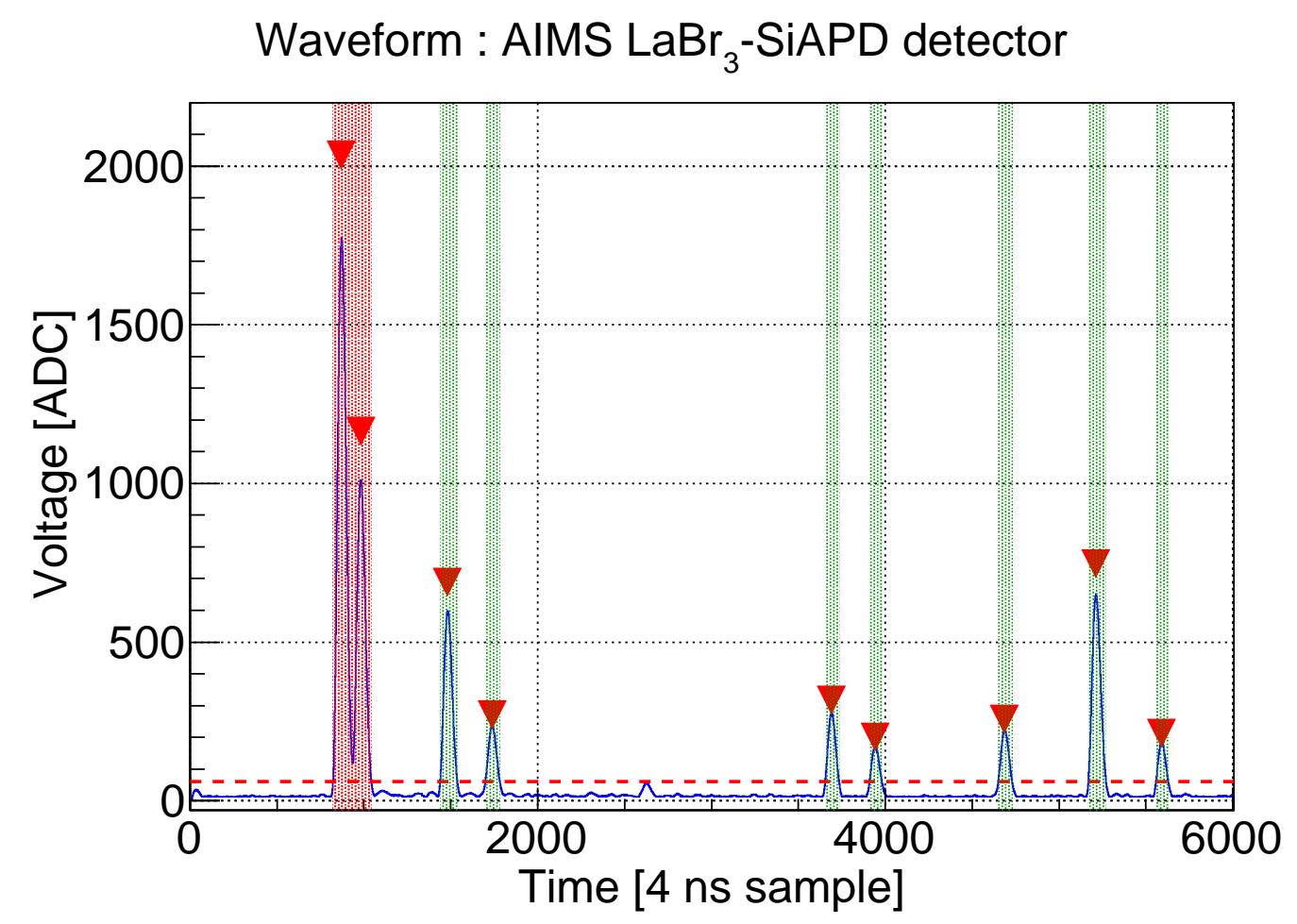

Figure 5: ADAQAnalysis demonstrating its multipeak finder algorithm, which robustly identifies all peaks (red triangles) above a floor (dotted red line), filters them with a pileup rejection algorithm (shaded green peaks are accepted; shaded red peaks are rejected), and then computes pulse height and area for spectroscopy. The data is from a lanthanumbromide scintillator coupled to a silicon avalanche photodiode and readout with a CAEN V1720 VME digitizer that is used in the AIMS diagnostic on the Alcator C-Mod magnetic fusion tokamak [7]. 


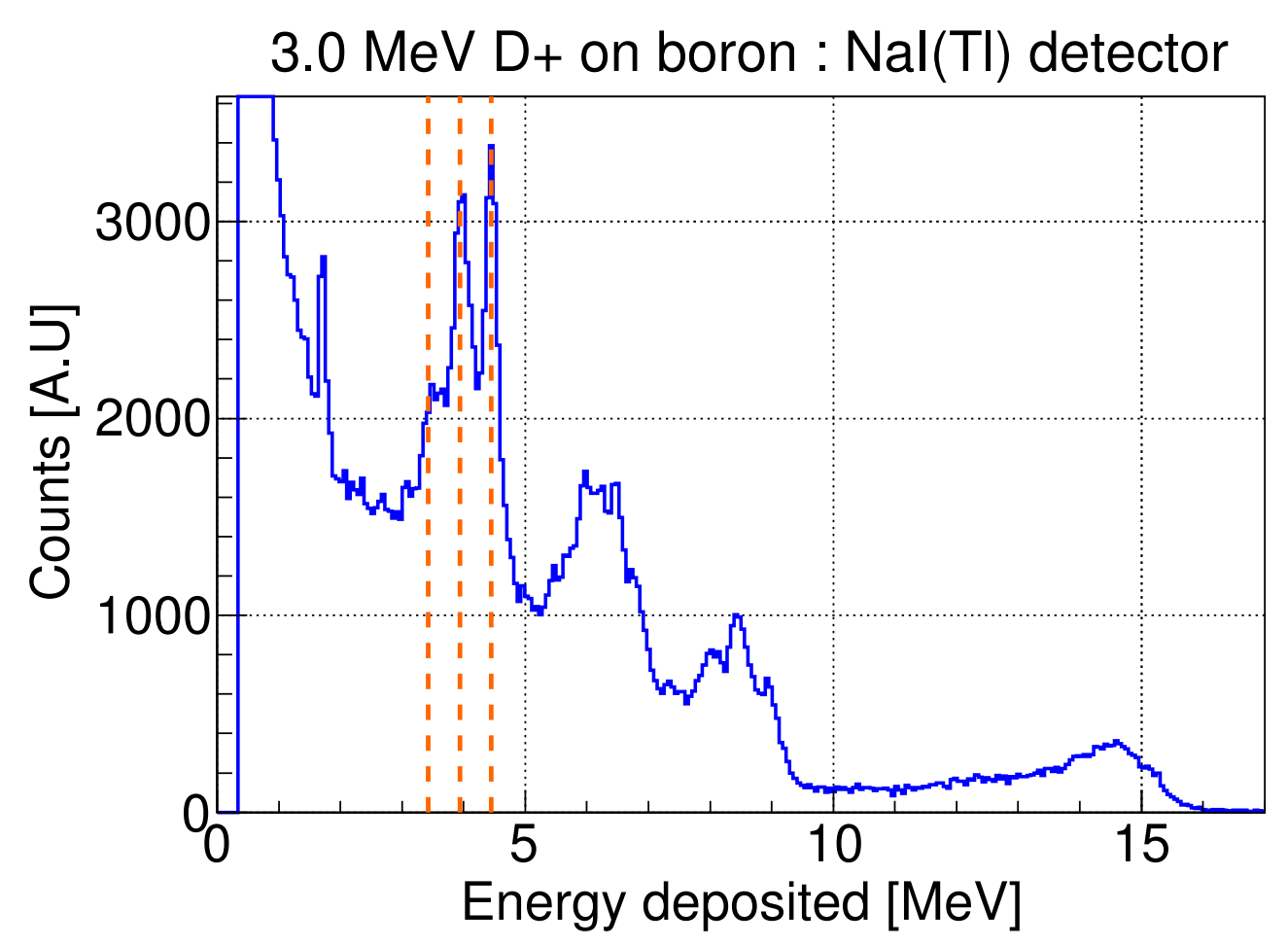

Figure 6: ADAQAnalysis locating the $4.44 \mathrm{MeV}$ full energy peak and first and second escape peaks in a gamma spectrum from $3.0 \mathrm{MeV}$ deuterons bombarding a natural boron target. The data was acquired with a $2^{\prime \prime} \times 4^{\prime \prime} \times 16^{\prime \prime} \mathrm{NaI}(\mathrm{Tl})$ scintillator coupled to a 2 -inch PMT and readout with a CAEN V1724 VME digitizer that is used in a monoenergetic gamma radiography experiment for nuclear security [8]. 
such as inorganic scintillators, high purity germanium, and charged particle - or by using an edge finding algorithm - suitable for detectors based on elastic scattering such as liquid organic scintillators. Once calibration points have been set, linear fits (for two or more calibration points) or quadratic fits (for three or more calibration points) can be used to create calibrate energy deposition spectra. Once a spectra is energy calibrated, energy analysis tools are available to the user, such as the escape peak finder shown locating the 4.44 MeV gamma and its escape peaks in the $\mathrm{NaI}(\mathrm{Tl})$ spectrum shown in Figure 6.

To obtain quantitative results, spectra can be further analyzed. Detailed spectra continua can be calculated using the powerful sensitive nonlinear iterative peak-clipping (SNIP) algorithm [11]. The user can tune a variety of algorithm parameters to get an excellent continuum across a complicated spectrum as shown in Figure 7, which shows a high purity germanium gamma spectrum for $1 \mathrm{MeV}$ protons bombarding a lithium-fluoride target. The continuum can then be subtracted to isolate the peaks, which can then be fit with a gaussian to obtain exact parameters. An integrator is available to analyze the unfitted or gaussian peaks as well as raw or continuum subtracted spectra.

\subsection{Pulse shape analysis and filtering}

Another primary feature of ADAQAnalysis is the capability to analyze the shape of digitized detector waveforms and create filters for determining which events are added to pulse spectra. Two widely used applications of this technique are PSD, in which the decay pulse timing in organic scintillators can be used to identify particles, and pileup rejection, which can greatly 


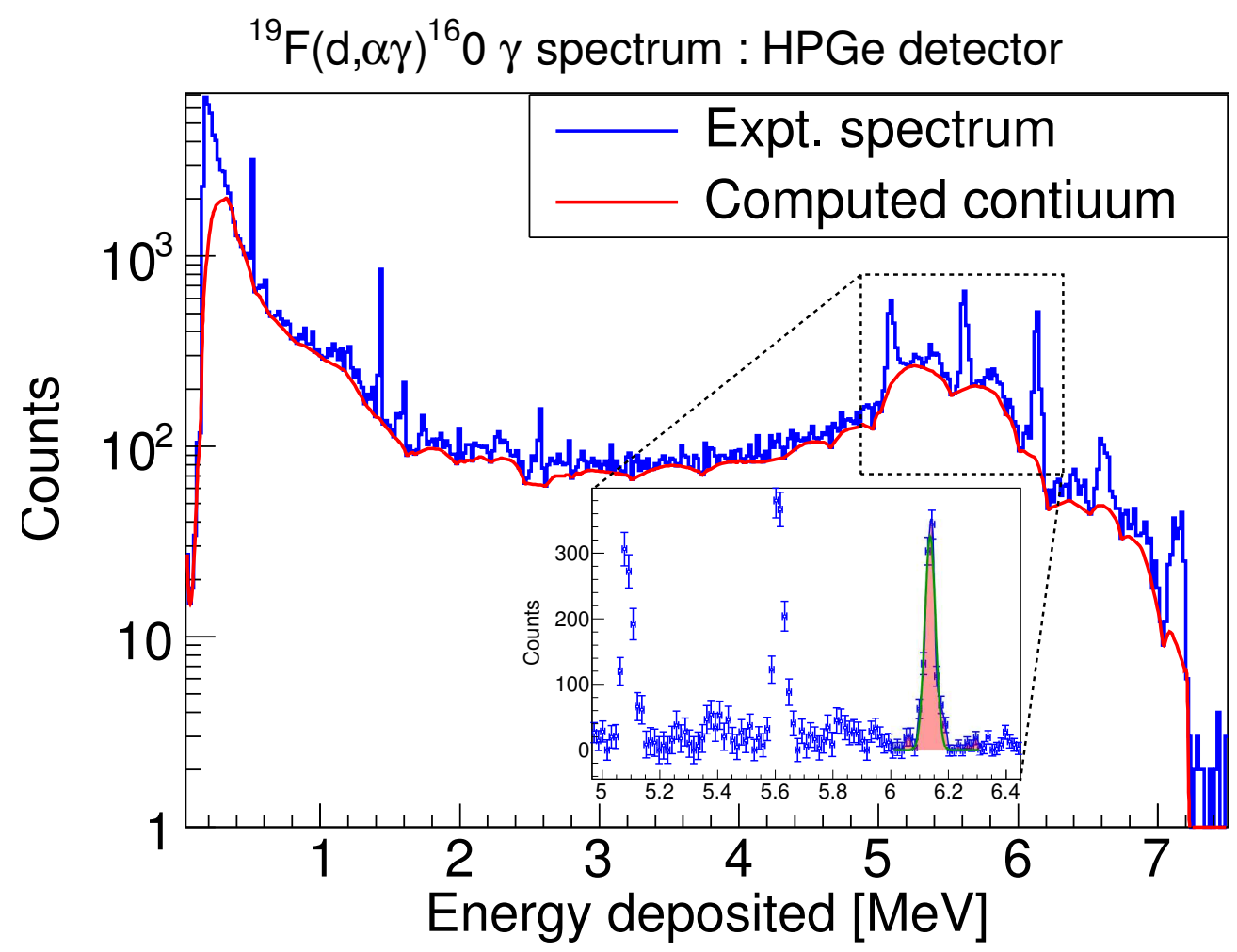

Figure 7: ADAQAnalysis accurately computing a gamma continuum for a high purity germanium (HPGe) detector spectrum; the inset shows a continuum-subtracted background with a gaussian fitted integral on the $6.1 \mathrm{MeV}$ peak. The continuum is computed using the sensitive nonlinear iterative peak-clipping (SNIP) algorithm within ROOT's TSpectrum class [11]. 
improve spectral features at high detector count rates.

ADAQAnalysis employs a digital version of the pulse shape analysis method known as the charge integral or dual gate method [12], in which an integral of the entire pulse - the "total" or "long" integral - is compared against a partial integral of the pulse, typically taken over the end of the pulse - the "tail" or "short" integral. When these integrals are binned into a 2D histogram, separation emerges between event groups of interest. An example is shown in Figure 8, in which well-separated fast neutron and gamma events from 1.1 MeV deuterons bombarding a natural boron target are measured with a EJ301 liquid organic scintillator.

Once a 2D histogram has been created, the user can create a graphical cut that will function as a filter for spectra creation, allowing the user to selectively include and exclude events based on their pulse shape, as shown by the magenta area surrounding the neutron events in Figure 8.

\subsection{Persistent storage and graphical output}

Finally, ADAQAnalysis is capable of saving almost all of the analysis objects - waveforms, spectra, continua, calibration, pulse shape histograms - as ROOT objects within a TFile or in ASCII or CSV formats for further analysis. At any time during the analysis workflow, the graphical canvas can be output in a variety of formats with the user able to specify a wide range of graphical attributes.

\section{Conclusion}

The ADAQ framework provides a comprehensive set of software tools that is designed to help scientists and engineers who use radiation detec- 


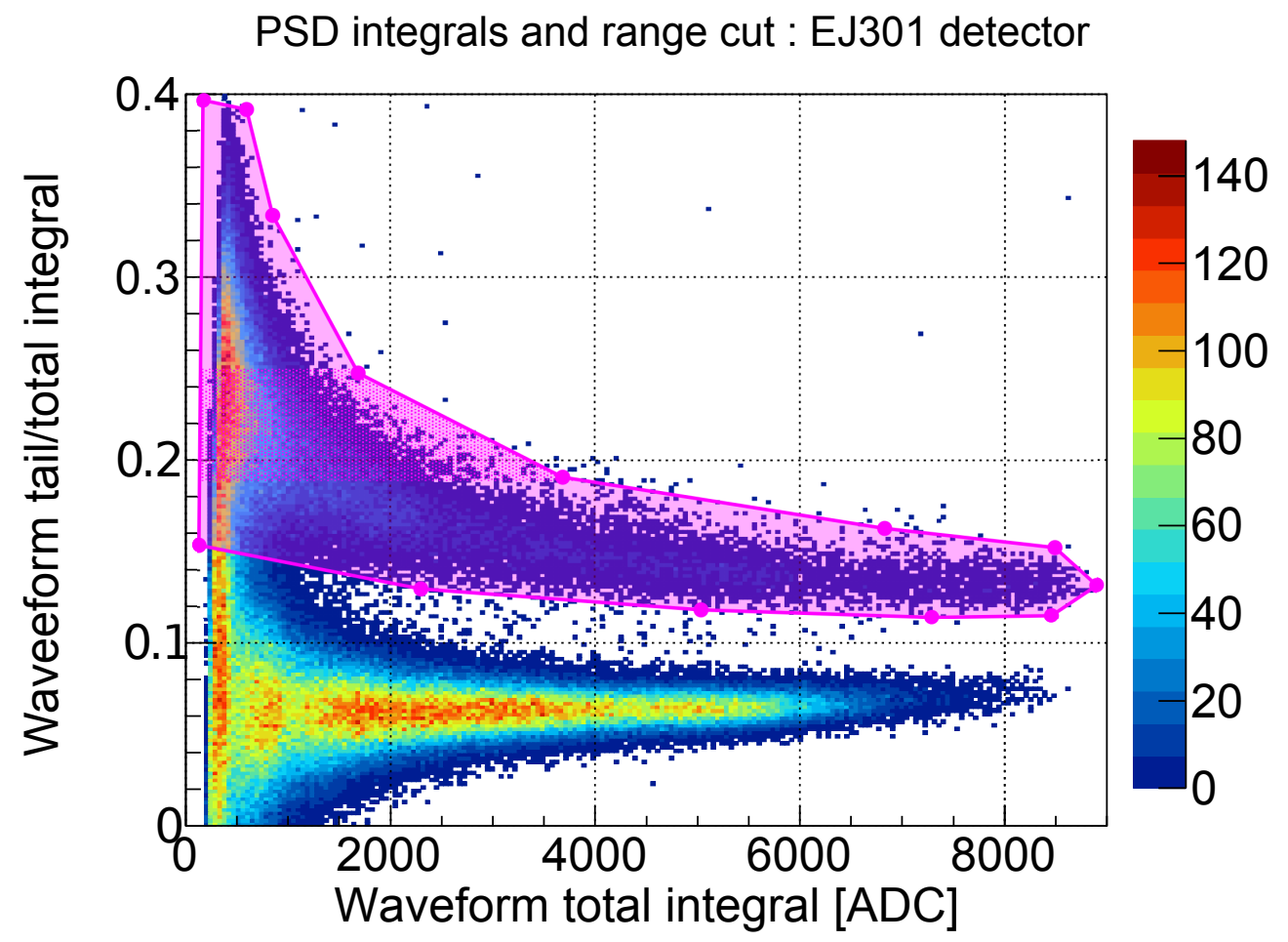

Figure 8: ADAQAnalysis demonstrating a graphical cut (magenta shaded region) on pulse shape discrimination data that can be used to determine which events are added to energy spectra. The data is from an EJ301 liquid organic scintillator detector measuring fast neutrons (top event bunch) and gammas (bottom event bunch) produced by a $1.1 \mathrm{MeV}$ deuteron beam bombarding a natural boron target. 
tors in their research more easily integrate the power of digital DAQs into their workflow. The framework is intended to be useful to all levels of user - from novice to expert - and provides a range of software from low-level development libraries to high-level GUI-based programs. The ultimate goal is enable users to maximize their research productivity by enabling their use of high performance digital DAQ hardware and handling a majority of their data acquisition and analysis needs. In addition, the ADAQ framework can be a powerful tool in the teaching laboratory in two ways: first, by allowing students to focus explicitly on learning modern detector pulse processing without hardware expertise or complicated electronic circuits; and second, by easily visualizing all steps of digital DAQ programming, data acquisition, and data analysis.

\section{Acknowledgments}

The author would like to thank Brandon Sorbom, Leigh Ann Kesler, Buck O'Day, Areg Danagoulian, TD MacDonald, Jill Rahon, Mareena Robinson, Gabrielle Ledoux (MIT), Mike Mayer, Jason Nattress (Penn State), and Paul Rose (Georgia Tech), who have served and continue to serve, sometimes painfully, as invaluable beta testers for the ADAQ framework. During development of the ADAQ framework, the author has been supported by U.S. DOE Grant DE-FG02-94ER54235, U.S. DOE Cooperative Agreement DE-FC02-99ER54512, and the US DOE Fusion Energy Sciences Postdoctoral Research Fellowship. 


\section{References}

[1] G. F. Knoll, Radiation Detection and Measurement, Wiley, 2000.

[2] R. Brun, F. Rademakers, ROOT an object oriented data analysis framework, Nuclear Instruments and Methods in Physics Research Section A: Accelerators, Spectrometers, Detectors and Associated Equipment 389 (1997) 81 - 86. New Computing Techniques in Physics Research.

[3] S. Agostinelli, J. Allison, K. Amako, J. Apostolakis, H. Araujo, P. Arce, M. Asai, D. Axen, S. Banerjee, G. Barrand, F. Behner, L. Bellagamba, J. Boudreau, L. Broglia, A. Brunengo, H. Burkhardt, S. Chauvie, J. Chuma, R. Chytracek, G. Cooperman, G. Cosmo, P. Degtyarenko, A. Dell'Acqua, G. Depaola, D. Dietrich, R. Enami, A. Feliciello, C. Ferguson, H. Fesefeldt, G. Folger, F. Foppiano, A. Forti, S. Garelli, S. Giani, R. Giannitrapani, D. Gibin, J. J. G. Cadenas, I. Gonzlez, G. G. Abril, G. Greeniaus, W. Greiner, V. Grichine, A. Grossheim, S. Guatelli, P. Gumplinger, R. Hamatsu, K. Hashimoto, H. Hasui, A. Heikkinen, A. Howard, V. Ivanchenko, A. Johnson, F. W. Jones, J. Kallenbach, N. Kanaya, M. Kawabata, Y. Kawabata, M. Kawaguti, S. Kelner, P. Kent, A. Kimura, T. Kodama, R. Kokoulin, M. Kossov, H. Kurashige, E. Lamanna, T. Lampn, V. Lara, V. Lefebure, F. Lei, M. Liendl, W. Lockman, F. Longo, S. Magni, M. Maire, E. Medernach, K. Minamimoto, P. M. de Freitas, Y. Morita, K. Murakami, M. Nagamatu, R. Nartallo, P. Nieminen, T. Nishimura, K. Ohtsubo, M. Okamura, S. O’Neale, Y. Oohata, K. Paech, J. Perl, A. Pfeiffer, M. G. Pia, F. Ranjard, A. Rybin, S. Sadilov, E. D. Salvo, G. Santin, T. Sasaki, N. Savvas, Y. Sawada, 
S. Scherer, S. Sei, V. Sirotenko, D. Smith, N. Starkov, H. Stoecker, J. Sulkimo, M. Takahata, S. Tanaka, E. Tcherniaev, E. S. Tehrani, M. Tropeano, P. Truscott, H. Uno, L. Urban, P. Urban, M. Verderi, A. Walkden, W. Wander, H. Weber, J. P. Wellisch, T. Wenaus, D. C. Williams, D. Wright, T. Yamada, H. Yoshida, D. Zschiesche, Geant4-a simulation toolkit, Nuclear Instruments and Methods in Physics Research Section A: Accelerators, Spectrometers, Detectors and Associated Equipment 506 (2003) $250-303$.

[4] The ADAQ software libraries: Available at https://github.com/zachhartwig/ADAQAcquisition, 2015.

[5] The ADAQAcquisition program: Available at https://github.com/zachhartwig/ADAQAcquisition, 2015.

[6] The ADAQAnalysis program: Available at https://github.com/zachhartwig/ADAQAnalysis, 2015.

[7] Z. S. Hartwig, H. S. Barnard, R. C. Lanza, B. N. Sorbom, P. W. Stahle, D. G. Whyte, An in situ accelerator-based diagnostic for plasmamaterial interactions science on magnetic fusion devices, Review of Scientific Instruments 84 (2013) - .

[8] Buckley E. O'Day III, A novel Low-dose Approach to Active Detection of Shielded High-Z Material, Ph.D. thesis, Massachusetts Institute of Technology, 2015.

[9] M. Mayer, J. Nattress, V. Kukharev, A. Foster, A. Meddeb, C. Trivelpiece, Z. Ounaies, I. Jovanovic, Development and characterization of a 
neutron detector based on a lithium glasspolymer composite, Nuclear Instruments and Methods in Physics Research Section A: Accelerators, Spectrometers, Detectors and Associated Equipment 785 (2015) 117 122.

[10] Z. S. Hartwig, P. Gumplinger, Simulating response functions and pulse shape discrimination for organic scintillation detectors with Geant4, Nuclear Instruments and Methods in Physics Research Section A: Accelerators, Spectrometers, Detectors and Associated Equipment 737 (2014) $155-162$.

[11] M. Morháć, J. Kliman, V. Matoušek, M. Veselský, I. Turzo, Efficient one- and two-dimensional gold deconvolution and its application to $\gamma$ ray spectra decomposition, Nuclear Instruments and Methods in Physics Research Section A: Accelerators, Spectrometers, Detectors and Associated Equipment 401 (1997) 385 - 408.

[12] F. Brooks, A scintillation counter with neutron and gamma-ray discriminators, Nuclear Instruments and Methods 4 (1959) 151 - 163. 